\title{
Pseudonym Creation and Parameter Based Key Generation for a Robust Authentication using ECC
}

\author{
${ }^{1} \mathrm{R}$ Bhakthavathsalam, ${ }^{2}$ Kavyashree U B, ${ }^{3}$ Maithri R Kumar, ${ }^{4}$ Nikhila Dwarakanath, ${ }^{5}$ Saripalli Pooja and \\ ${ }^{6}$ Gowranga K H \\ ${ }^{1,6}$ Supercomputer Education and Research Centre, Indian Institute of Science, Bangalore, India; \\ 2, 3, 4, 5 Dayananda Sagar College of Engineering, Bangalore, India; \\ bhaktha@serc.iisc.ernet.in; kavyashree.ub9294@gmail.com; maithri.rk@gmail.com; \\ nikhiladknath@gmail.com; poojasaripalli@gmail.com; gowranga@cds.iisc.ac.in
}

\begin{abstract}
Security is an important aspect in every field today and it cannot be neglected whatsoever. Network security has become one of the most important concerns of this generation due to its wide range of applications. With the emerging techniques in elliptic curve cryptography (ECC), it has now become the mainframe of many cryptosystems. Due to the generation of small key sizes in ECC, it poses as a favourable cryptosystem that can be used to minimize memory consumption. The previous schemes that were developed were unable to achieve user anonymity and were also vulnerable to stolen-verifier attacks, offline password guessing and insider attacks. The proposed scheme aims at improving the security provided to a user during authentication phase involved in any transaction. A step-by-step process is outlined in order to enhance security. It intends to provide a system involving Elliptic curve cryptography along with One Time Passwords to implement a new authentication scheme that overcomes certain threats to network security. The main features of this scheme includes generation of a common key with scalar multiplication, provision of user anonymity in order to prevent man-in-the-middle attacks using the Elliptic curve base point and a random number in order to prevent session hijacking attack or replay attack. This authentication paradigm finds its applications in ATM systems, RFID tags, smart card applications, mobile applications with SIM cards and so on. The proposed scheme works favourably with devices that have less resources (like memory).
\end{abstract}

Keywords: Elliptic curve cryptography (ECC); User anonymity; One Time Password (OTP); Network security; Scalar multiplication; Authentication scheme; Decryption; Encryption; Key agreement; Key generation.

\section{Introduction}

Information security [1] is the application of measures to ensure the safety and privacy of data by managing its storage and distribution. Information security has both technical and social implications. The first simply deals with the 'how' and 'how much' question of applying secure measures at a reasonable cost. The second grapples with issues of individual freedom, public concerns, legal standards and how the need for privacy intersects them. This discussion covers a range of options open to business managers, system planners and programmers that will contribute to your ultimate security strategy. The eventual 
choice rests with the system designer and issues. Modern security products are now designed to balance the needs of business on the Internet while protecting against today's sophisticated threats. Modern information security practices have evolved into a blended approach to managing access to information.

In implementing a security system, all data networks deal with the following main elements:

- Hardware which includes servers, redundant mass storage devices, communication channels and lines, hardware tokens (smart cards) and remotely located devices (e.g., thin clients or Internet appliances) serves as interfaces between users and computers

- Software, includes operating systems, database management systems, communication and security application programs

- Data, including databases containing customer - related information.

- Personnel, are originators or/and users of data and they may be professional personnel, clerical staff, administrative personnel, and computer staff.

Cryptography [1] is the method of converting data from a human readable form to a modified form, and then back to its original readable form, to make unauthorized access difficult. Cryptography is used in the following ways:

- provides data security by ensuring adequate encryption of the data.

- It provides a means to check if data has been manipulated or not, thus ensuring data integrity.

- provides data uniqueness by confirming that data is "original", and not a "duplicate". The "original" data is sent with a unique identifier. This unique identifier is then checked by the receiver of the data.

\subsection{Elliptic Curve Cryptography}

Elliptic curve cryptography (ECC) [2] is a public key encryption technique based on the concept of elliptic curves that has gained popularity recently in creating cryptographic keys. Elliptic curve cryptography uses the properties of a basic elliptic curve equation for the key generation over the generation of the product of very large prime numbers. It can be used in conjunction with most public key encryption methods, such as RSA, and Diffie-Hellman. According to some researchers, ECC can yield a level of security with a 160-bit key that other systems require a 1,024-bit key to achieve. Fig 2 shows a comparison between the key sizes generated by RSA and ECC. The equation of an elliptic curve is: $y^{2}=x^{3}+a x+b(1)$, where $a$ and $b$ must satisfy the condition $4 a^{3}+27 b^{2} \neq 0$ (2). There are two basic group operations known as Prime Field Arithmetic operation and Scalar Multiplication performed on elliptic curves as explained below:

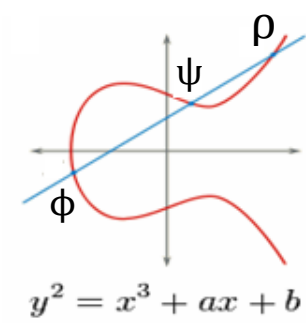

Figure 1: Elliptic curve [6]

\begin{tabular}{|c|c|}
\hline ECC(in bits) & RSA (in bits) \\
\hline 106 & 512 \\
\hline 112 & 768 \\
\hline 132 & 1024 \\
\hline 160 & 2048 \\
\hline 283 & 7680 \\
\hline
\end{tabular}

Figure 2: Comparison of key sizes in RSA and ECC [7] 
R Bhakthavathsalam, Kavyashree U B, Maithri R Kumar, Nikhila Dwarakanath, Saripalli Pooja and Gowranga K H; Pseudonym Creation and Parameter Based Key Generation for a Robust Authentication using ECC, Transactions on Networks and Communications, Volume 4 No. 3, June (2016); pp: 34-44

\subsection{Prime Field Arithmetic}

The prime field GF(p) [3] is the finite field whose elements are all the integers between 0 and $p-1$ inclusive. For every a $€ \mathrm{GF}(\mathrm{p})$ the following operations are defined as:

- Addition i.e., If $a, b € G F(p)$ then $a+b=r$, where $r € G F(p)$ and equals $(a+b) \bmod p$.

$\circ$ Multiplication i.e., If $a, b \in G F(p)$ then $a * b=r$, where $r € G F(p)$ and equals $(a * b) \bmod p$.

$\circ$ Inversion i.e., If $a$ is a non-zero element in $\mathrm{GF}(p)$, then $a^{\prime} 1=\mathrm{c} € \mathrm{GF}(\mathrm{p})$ such that $a^{*} c=1 \bmod p$.

Points whose coordinates $(x, y)$ satisfy equation along with the point of infinity $P \infty$ (which is at the top of the $y$-axis), make up the set of rational points on curve E W. Trappe and L. Washington (2002). The negative of a point $P$ is its reflection in the $x$-axis, i.e., $-P=(x,-y)$. For elliptic curves over prime fields $G F(p)$ with $p>3$, the parameters $a$ and $b$ of eq. 2 should satisfy the condition $4 a 3+27 b 2 \neq 0(\bmod p)$. D Hankerson et al. (2004). The condition is required to ensure that the curve is smooth, and there are no points at which the curve has two or more distinct tangent lines. The allowable point coordinates become integer modulo p. The standard representation of points on an elliptic curve E over prime fields is the Affine coordinates. The graphical representation of point addition is computed using Eq.3, Eq. 4 and Eq. 5 and the graphical representation of point doubling is computed using Eq.6, Eq.7 and Eq.8.

$$
\begin{aligned}
& \lambda=\left(Y_{2}-Y_{1}\right) *\left(X_{2}-X_{1}\right)-1 \\
& X_{3}=\lambda_{2}-X_{1}-X_{2} \\
& Y_{3}=\lambda\left(X_{1}-X_{3}\right)-Y_{1} \\
& \lambda=\left(3 X_{1}^{2}+a\right) *\left(2 Y_{1}\right)-1 \\
& X_{3}=\lambda_{2}-2 X_{1} \\
& Y_{3}=\lambda\left(X_{1}-X_{3}\right)-Y_{1}
\end{aligned}
$$

Point multiplication, or scalar multiplication, is higher in the hierarchy of ECC operations than the point addition and doubling. It is computed by performing a series of point additions and point doublings. A point ' $P$ ' is multiplied by a scalar $k$ to yield the point ' $k P$ '. For example, to compute ' $5 P$ ' one would double ' $\mathrm{P}$ ' twice to obtain ' $4 \mathrm{P}$ ' then add ' $\mathrm{P}$ ' to the intermediate result to obtain ' $5 \mathrm{P}$ '. Various algorithms exist for point multiplication. When the point $P$ is unknown the unknown point multiplication is used, whereas when the point is fixed, the known point multiplication is used.

The known point multiplication is faster than the unknown point multiplication because certain fixed parameters are known. When two points are added or doubled in Affine Coordinates I. Blake et al. (1999), we need to perform modular inversion to compute p, e.g., (X2-X1)-1 or (2Y1)-1, in eq.3 and eq.6. Modular inversion is a computationally intensive operation, and it is required each time a point is added or doubled. There are alternative point representations that favour modular multiplications over inversions. Some modular operations are typically required in a larger framework such as the signature schemes.

The performance of field multiplication is fundamental to mechanisms based on elliptic curves. Constraints on hardware integer multipliers and the cost of carry propagation can result in significant bottlenecks in direct implementations of algorithms. 


\section{Related Works}

\subsection{Islam Biswas Scheme:}

Islam and Biswas [4] proposed an advanced password authentication scheme based on ECC. The authors claimed that their scheme provides mutual authentication and is free from all known cryptographic attacks, such as replay attack, offline password guessing attack, insider attack and so on. Although their scheme is superior to the previous solutions for implementation on mobile devices, we find their scheme cannot achieve the claimed security: their scheme is vulnerable to the offline password guessing attack, the stolen verifier attack.

Islam-Biswas's scheme consists of four phases: the registration phase, the authentication phase, the session key distribution phase and the password change phase. In Islam-Biswas's scheme, the user's identity ID is transmitted in plain, which may leak the identity of the logging user once the login messages were eavesdropped. In a word, neither initiator anonymity nor initiator un-traceability can be preserved in their scheme.

\subsection{Chun Ta Li's Scheme:}

The Chun Ta Li's scheme [5] for user authentication was an enhancement from the other schemes that were already provided. It is demonstrated that the so-called secure, anonymous user authentication scheme introduced by He et al. is vulnerable to eavesdropping attack and is not practical for real-life implementation. Li showed that user anonymity of their scheme is not achieved, the user has to bear in mind a long identity (128 bit) during the login phase, and there is no provision for fairness in the key agreement.

To remedy these security weaknesses, Li further proposed a novel authentication scheme which is immune to various known types of attack and is more secure and practical for mobile wireless networking. However, it is found that Li's authentication scheme has a serious security problem in that all registered users' sensitive passwords can be easily derived by the privileged-insider of remote server. The attack exists because the data written on smart card is enough to compute the password.

\section{System Model}

A user initially registers himself/herself with the server by providing his PIN (Personal Identification Number) and password via the client system. A secure communication channel is established between the client and the server before the information is sent in an encrypted format. The server stores the password verifier and pseudonym of the user's PIN in the verifier table after performing decryption. Upon further accesses, the authentication phase takes place and only if the right password and OTP are entered, the user is granted permission to login. The user is given a maximum of three trials. The OTP is sent to the user's registered email address. The established channel is used in every phase to communicate in a secure manner.

The user, client and server pose as active participants in every phase. A change of password upon request is provided to the user. An additional option of account deletion is provided in case the user wishes to deactivate and delete his/her account. On choosing account deletion, both the client and server systems authenticate the user details and perform the deletion operation and simultaneously update the same in their respective systems. Else, a status bit of 0 is sent indicating that the user does not wish to delete his account. 


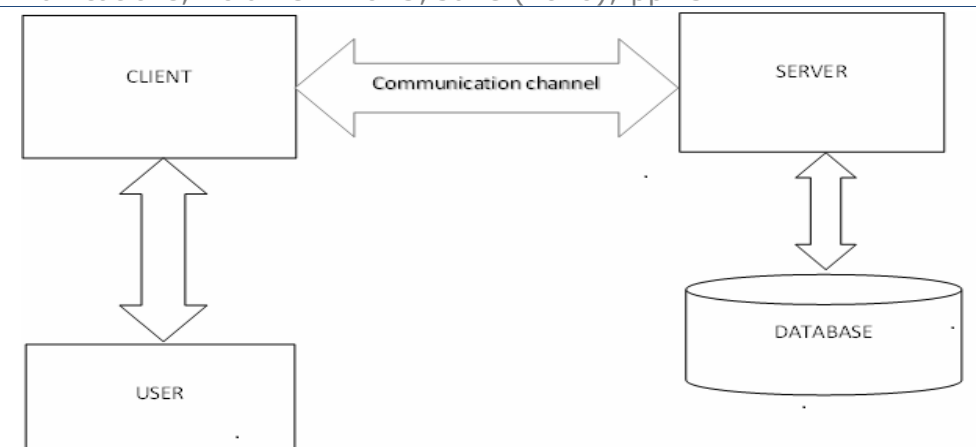

Figure 3: Block Diagram for System Design Model

\section{A Secure Authentication Algorithm}

The algorithms [5] aim at providing layers of security to the system to prevent security attacks like replay attack, session hijacking and man-in-the-middle attack. Security provided by this scheme is robust and efficient. The proposed scheme consists of four phases namely registration phase, authentication phase, password change phase and user eviction phase. Now each of these phases is described. The following are the terminologies that are going to be used throughout the paper:

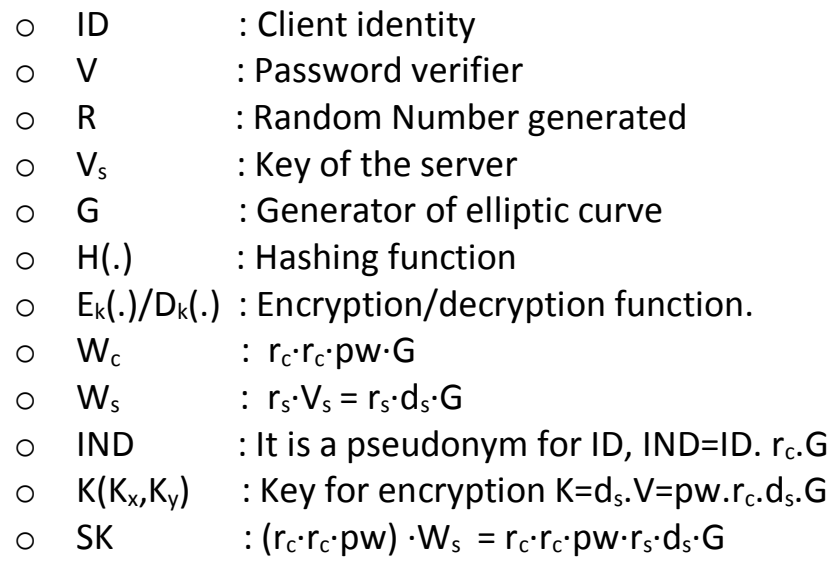

\subsection{Registration Phase:}

When a client [5] wants to become a new legal client, the client must register to the server with his/her PIN ID, password pw and Email ID. Then server stores each legal client's identity IND (pseudonym of user's identity, as computed on client side), password-verifier V, a status-bit, the Email ID and the flag bit, where the status-bit indicates the login status of the client to prevent many logged-in users' attack. If the client is logged in to the server, the status-bit is set to 1 ; otherwise it is set to 0 . The flag bit is set to 0 . Upon each unsuccessful attempt, the flag is incremented; if flag equals 3 , access is denied.

\subsection{Authentication Phase:}

When a client [5] wants to access the Server, the client has to enter the PIN ID and password. This phase mainly involves validating the user and also provides an option for sending an OTP for a more secure authentication. The client then computes IND. The following steps are then performed: 
Step 1:User $\rightarrow$ Server: IND, $E_{K x}\left(I N D, R, W_{c}, V^{\prime}\right)$

The client retrieves $r_{c}$, generates a new random number $r_{c}{ }^{\prime}$, computes

$R=r_{c} \cdot V_{s}=r_{c} \cdot d_{s} \cdot G, W_{c}=r_{c} \cdot r_{c} \cdot p w \cdot G, V^{\prime}=p w \cdot r_{c}{ }^{\prime} \cdot G$ and $E_{k x}\left(I N D, R, W_{c}, V^{\prime}\right)$ and sends ID and $E_{k x}\left(I N D, R, W_{c}, V^{\prime}\right)$ to Server, where $V_{s}$ is the public key of Server and encryption key $K_{x}$ is the $x$ coordinate of $K=p w . r_{c} . V_{s}=$ pw. $\mathrm{r}_{\mathrm{c}} \cdot \mathrm{d}_{\mathrm{s}} \cdot \mathrm{G}=\left(\mathrm{K}_{\mathrm{x}}, \mathrm{K}_{\mathrm{y}}\right)$.

Step 2: Server $\rightarrow$ User: $\left(W_{c}+W_{s}\right), H\left(W_{s}, V^{\prime}, S K\right)$

Server computes the decryption key $K_{x}$ by computing $K=d_{s} \cdot V=p w \cdot r_{a} \cdot d_{s} \cdot G=\left(K_{x}, K_{y}\right)$ and decrypts $E_{K x}$ (IND, $\left.R, W_{c}, V^{\prime}\right)$ to reveal (IND, $\left.R, W_{c}, V^{\prime}\right)$. Then Server compares decrypted ID with received IND . If all the conditions are satisfied, Server sends $\left(W_{c}+W_{s}\right)$ and $H\left(W_{s}, V^{\prime}\right)$ to client, where $W_{s}=r_{s} \cdot V_{s}=r_{s} \cdot d_{s} \cdot G$ and $r_{s}$ is a random number which is generated by Server. Server generates the session key

$S K=\left(r_{s} \cdot d_{s}\right) \cdot W_{c}=r_{c} \cdot d_{s} \cdot p W \cdot r_{c} \cdot r_{c} \cdot G$.

\section{Step 3: User $\rightarrow$ Server: IND ', $H\left(W_{c}, W_{s}, V^{\prime}, S K\right)$}

Client retrieves $W_{s}$ by subtracting $W$ from $\left(W_{c}+W_{s}\right)$ and checks whether the hashed result of $\left(W_{s}, V^{\prime}, S K\right)$ is equal to the received $\mathrm{H}\left(\mathrm{W}_{s}, \mathrm{~V}^{\prime}, \mathrm{SK}\right)$. If so, the client computes $\mathrm{H}\left(\mathrm{W}_{\mathrm{c}}, \mathrm{W}_{\mathrm{s}}, \mathrm{V}^{\prime}, \mathrm{SK}\right)$ and sends it to Server. Here, the client computes the final session key $S K=\left(r_{c} \cdot r_{c} \cdot p w\right) \cdot W_{s}=r_{c} \cdot r_{c} \cdot p w \cdot r_{s} \cdot d_{s} \cdot G$. The client also generates the new pseudonym IND'= ID. $r_{c}{ }^{\prime} . G$ and sends it to the Server.

\section{Step 4: Server $\rightarrow$ User: Access Granted/Denied}

Server uses its own $W_{s}$ and $\left(W_{c}, V^{\prime}\right)$ which is received from client in Step 1 to compute $H\left(W_{c}, W_{s}, V^{\prime}, S K\right)$ and checks whether the hashed result of $\left(W_{c}, W_{s}, V^{\prime}, S K\right)$ is equal to the received $H\left(W_{c}, W_{s}, V^{\prime}, S K\right)$. If it holds, then Server generates an OTP and sends it to the Email ID the user registered with. Only is the user enters the right OTP within the given amount of time ,Server grants login request and replaces old password-verifier $V=p w \cdot r_{c} \cdot G$ with new password-verifier $V^{\prime}=p w \cdot r_{c}{ }^{\prime} \cdot G$, and the old pseudonym IND=ID. $r_{c} \cdot G$ by the new pseudonym IND'=ID. $r_{c}{ }^{\prime}$.G ,otherwise Server denies login request. Finally, the user replaces $r_{c}$ with $r_{c}^{\prime}$ if all of the conditions are satisfied. After finishing the authentication phase, the verifier table is updated.

\subsection{Password change Phase:}

When the client [5] wants to change his/her original password pw to a new password pw', client must notify the server to update the old password-verifier $V=p w \cdot r_{c} \cdot G$ to a new password-verifier $V^{\prime}=p w^{\prime} \cdot r_{c}^{\prime} \cdot G$. If the authentication token $\mathrm{H}\left(\mathrm{W}_{c}, \mathrm{~W}_{\mathrm{s}}^{\prime}\right)$ is valid,Server subtracts $\mathrm{W}_{c}$ from $\mathrm{W}_{\mathrm{c}}+\mathrm{V}^{\prime}$ to reveal new passwordverifier $V^{\prime}$ and replaces $V$ with $V^{\prime}$ if the computed value of the hash function of $\left(W_{s}, V^{\prime}\right)$ is the same as the received value of the hashed result of $\left(W_{s}^{\prime}, V^{\prime}\right)$. The steps involved in the password change phase are as follows:

O Step 1: User $\rightarrow$ Server: IND, $E_{K x}\left(I N D, R, W_{c}, V^{\prime}\right)$

- Step 2: Server $\rightarrow$ User: $\left(W_{c}+W_{s}\right), H\left(W_{s}, V^{\prime}\right)$

- Step 3: User $\rightarrow$ Server: IND', $H\left(W_{c}, W_{s}, V^{\prime}, S K\right), H\left(W_{s}+W_{c}+V^{\prime}\right)$

$\circ$ Step 4: Server $\rightarrow$ User: Password Change Granted/Denied

\subsection{User Account Deletion Phase:}

After the user [5] has been successfully authenticated, the user can choose to delete the registered account. Upon deletion of account, the user's details are deleted on the client side. The client then communicates with the server to do the same. The server deletes the user's details from the verifier table. The same user cannot further obtain access after account deletion. 


\section{Implementation Analysis}

The following are the steps involved during the execution of the algorithm:

- The user enters his/her option for registration, authentication or password change.

- During registration, the end-result is a successful registration of the user, with the details of the user being stored on the server verifier table.

- Upon authentication, the end-result is either access granted or denied during a transaction.

- If the user wishes to update the old password to a new password, a password change option is available.

- Finally, an option of deletion of the user's account is available, which deletes the user's information stored from the server and client records.

\section{Testing and Results}

Firstly, the user has to select the phase that he wishes to execute.

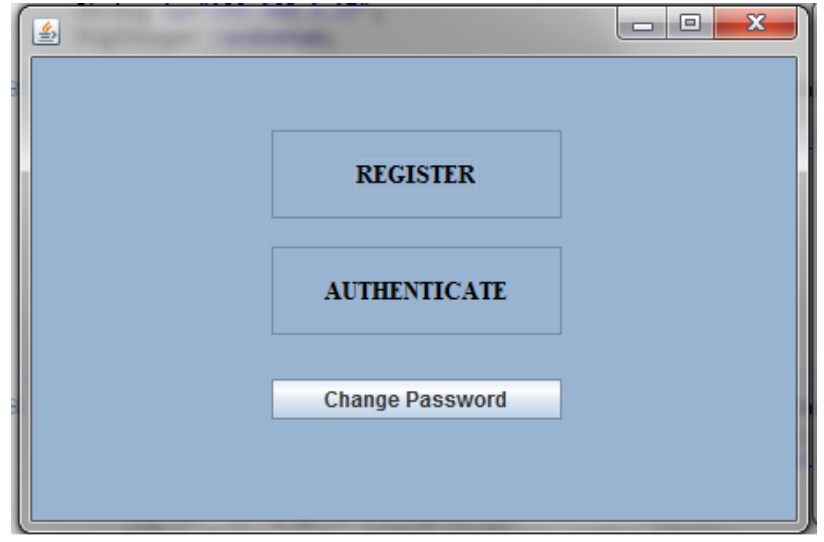

Figure 4: User's choice

\section{Case 1: Registration phase}

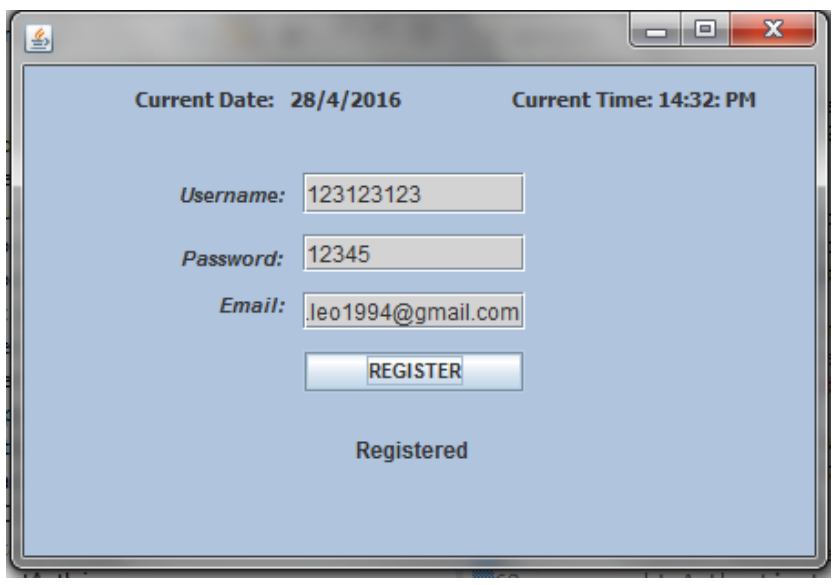

Figure 5: User Registration

At the client side, the user registers himself with his user PIN, password and email. The values received at server side are the base point (G) on EC curve and public key (Us) of server. 
Gx value: 48439561293906451759052585252797914202762949526041747995844080717082404635286

Gy value: 36134250956749795798585127919587881956611106672985015071877198253568414405109

Usx value: 36596164505861719536891134875938286035269234397048407665647988384691841560546

Usy value: 93205104400563970105249703998507405912734026643860219403854250994200355396440

Registered

Figure 6: User Registration process

Figure 6 represents successful registration. Upon successful registration, public key of the server(Us) and generator point $(\mathrm{G})$ are sent to the client.

\section{Server Side:}

in servereg

this is first

Recieved values:

Id: $65610 f 06430 c 44 f f d 47 f 9758 d c c 039 a a f 237 a 48 a 761 e 087 f d 19 c b 89 b 21189 b d c$

Uax: 114389246539511297889434555147771680178483245941849016691921419212838168355050

Uay: 91263717630602301126003006923363115127728376209826875349924634182123550097048

email id: motz.leo1994@gmail.com

sent Gx

sent Gy

sent Us:x

sent Usy

Successfully Registered

Figure 7: Server side successful registration

\section{Case 2: Authentication phase}

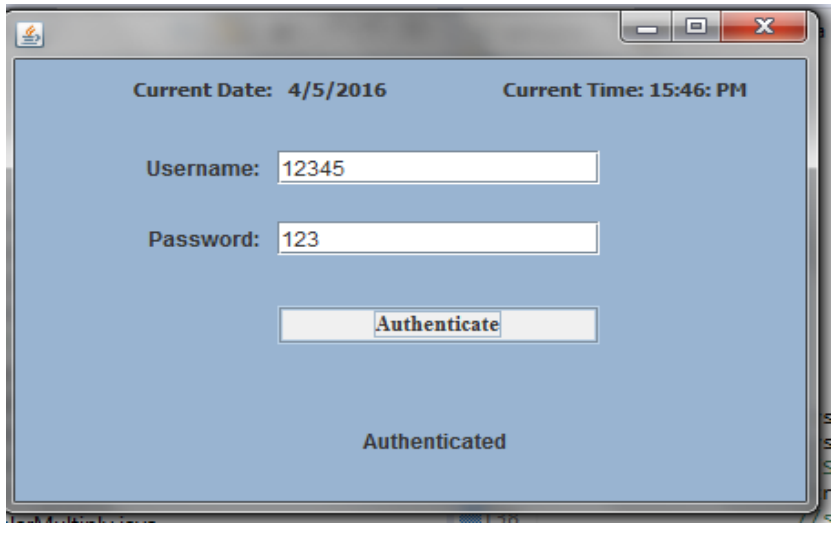

Figure 8: User authentication

During this phase, user enters the registered user PIN and password. Results of necessary computations are exchanged between client and server. For verification purposes an OTP is sent to the email id of the user. The user is required to enter this at the client side which is sent to the server.

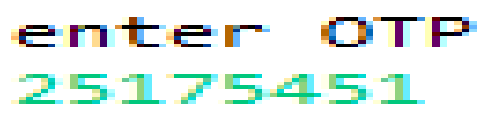

Figure 9: OTP entry

On successful authentication, the user can choose to delete his account. 
R Bhakthavathsalam, Kavyashree U B, Maithri R Kumar, Nikhila Dwarakanath, Saripalli Pooja and Gowranga K H; Pseudonym Creation and Parameter Based Key Generation for a Robust Authentication using ECC, Transactions on Networks and Communications, Volume 4 No. 3, June (2016); pp: 34-44

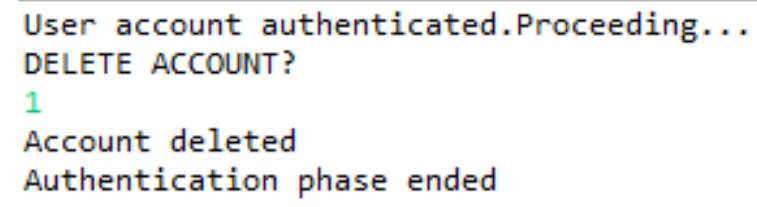

Figure 10: Successful authentication

\section{Server side:}

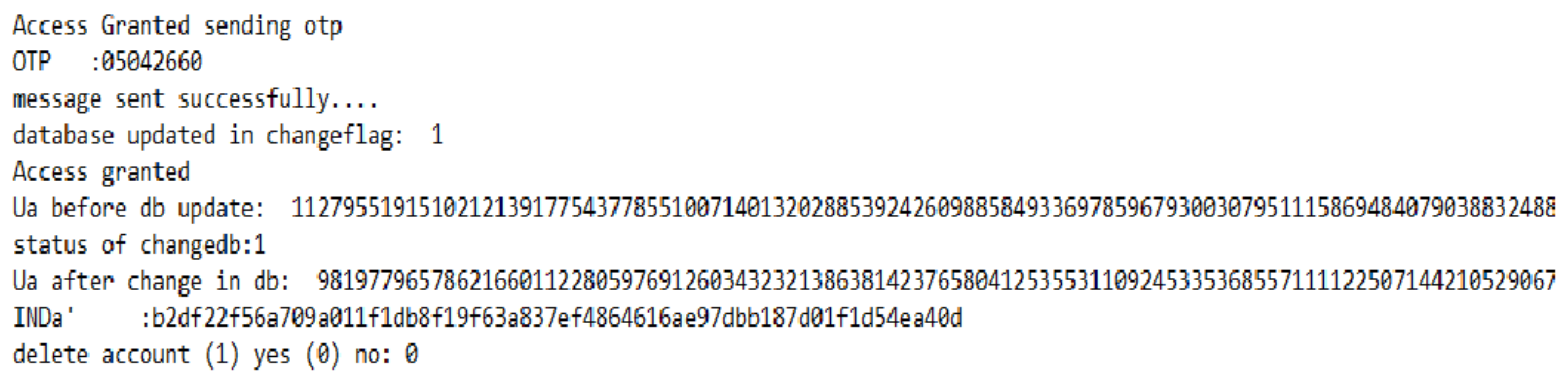

Figure 11: Result for successful authentication

Access Granted sending otp

OTP : :53191471

message sent successfully....

database updated in changeflag: 1

Access granted

Ua before db update: $\quad 510651189819669862568676214361685285328804089414846289653291019837209718680046240002876437296482340004$

status of changedb:1

Ua after change in $\mathrm{db}: \quad 64786553625703015227756294071471747728809586583462891259787403513096367580424263302901432602013873$

INDa' :0e2a184e0ed998c60bee3a5814f6e256f9a1cefbc6e3f146aeba58b19b49cfe7

delete account (1) yes (0) no: 1

database updated: 1

Figure 12: Result for account deletion

The user has a choice of deleting his account only after authentication, when he no more requires it. Fig 11 and Fig 12 show the user's choice to delete account. Fig 12 depicts deleting account, whereas Fig 11 depicts only authentication. In authentication phase, 1st the check for correct password is done, only then an OTP is mailed to the user. Upon the right entry, he is given an option of deleting account. If he chooses 1 , then account is deleted.

\section{Case 3: Password change phase}

During this phase, the user who wishes to change his password, has to enter his old password and also enter the new password and notifies the server to perform the same. After several steps of verification, as described in the proposed scheme, the password is changed. This step, too, includes the user entering OTP. The server then authenticates the user, only if the user has entered the correct password, he is allowed to change it. The server then saves the new password verifier in place of the old verifier against the username specified. Upon successful change, an appropriate status is reflected. 


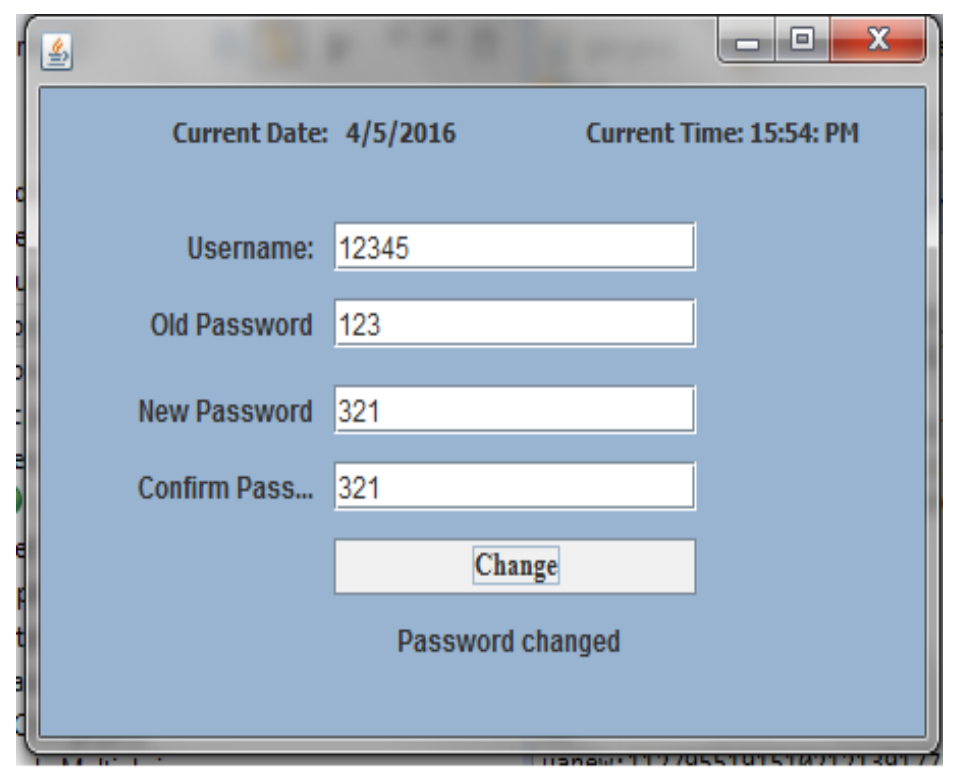

Figure 13: User password change

\section{Server side:}

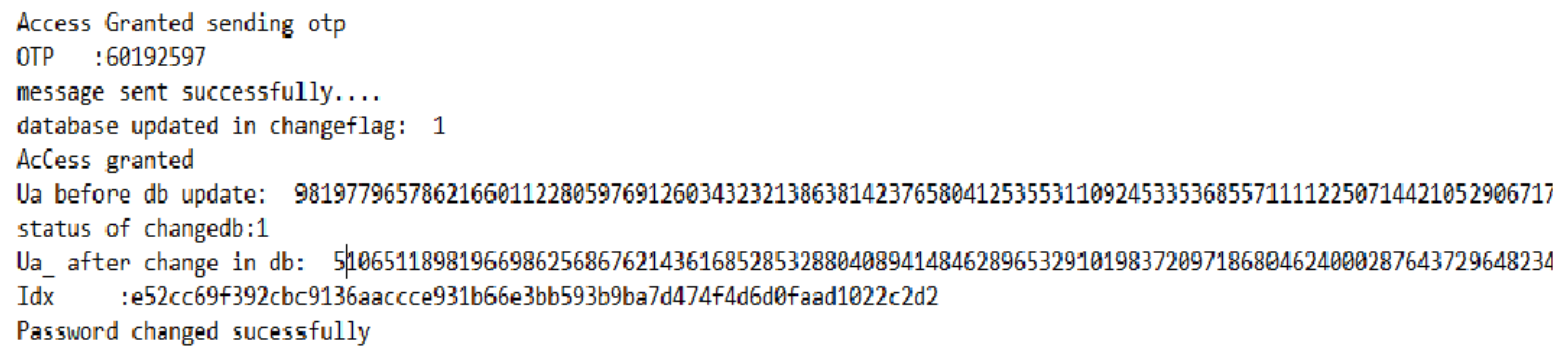

Figure 14: Result for password change

When the user wants to change his password, he is first authenticated as mentioned previously and then granted the permission for change his password.

\section{Conclusion}

An advanced password authentication and updated scheme is implemented that empowers login clients and remote servers with a secure and privacy-preserving authentication. Even though the passwordverifier is compromised by an adversary, the system does not worry that the stolen password-verifier will be used by others. Moreover, the proposed scheme allows a registered client to login to the server anonymously, when a pseudonym is used during the authentication procedure. Therefore, the proposed ECC-based scheme achieves mutual authentication, lower computation cost [8] and privacy protection. This scheme is an efficient and robust alternative for other popular schemes that use RSA cryptographic algorithm. It attempts to overcome a variety of drawbacks present in previous schemes.

Since the mechanism of parameter-based key generation is different on both client and server, and uses different number of parameters for the creation of keys, this scheme presents a unique way for the key generation protocol. Another major observation here is that the PIN that is provided by the user changes during every session, which makes it more secure to attacks by outsiders. The password provided by the user is not sent directly; instead, it is modified using elliptic curve parameters to form a password verifier 
R Bhakthavathsalam, Kavyashree U B, Maithri R Kumar, Nikhila Dwarakanath, Saripalli Pooja and Gowranga K H; Pseudonym Creation and Parameter Based Key Generation for a Robust Authentication using ECC, Transactions on Networks and Communications, Volume 4 No. 3, June (2016); pp: 34-44

which changes every session. Additional features like account deletion and usage of OTP makes this scheme more user-friendly and efficient.

This scheme can be effectively implemented in the login portals, online payment application services (like Bitcoin) that are prevalent in a variety of applications in today's technology, which can be altered to suit each application's requirements.

To further enhance security, biometric features like fingerprint verification, retinal detection and verification can be added which makes it more robust, secure and efficient. QR code can be used at the time of authentication. This scheme can be extended on to smart cards, RFID tags, SIM cards and other mobile applications.

\section{REFERENCES}

[1] The concept of Security: www.smartcardbasics.com/smart-card- security.html

[2] A brief introduction to Elliptic Curve Cryptography and the algorithms: http://searchsecurity.techtarget.com/definition/elliptical-curve- cryptography

[3] Prime Field Arithmetic: http://andrea.corbellini.name/2015/05/23/elliptic-curve-cryptography-finitefields-and-discrete-logarithms/

[4] Islam Biswas Scheme for User Authentication: https://eprint.iacr.org/2012/190.pdf

[5] Chun-Ta Li: A new password authentication and user anonymity scheme based on elliptic curve cryptography and smart card, IET Inf. Secur, 2013, Vol. 7, Iss 1.

[6] William Stallings: Cryptography and Network Security, 3rd edition, Published November 16th 2005 by Prentice Hall Hardcover, 680 pages.

[7] Bos, Joppe W, et al. Elliptic curve cryptography in practice; Financial Cryptography and Data Security, Springer Berlin Heidelberg, 2014, 157-175.

[8] Sukalyan Goswami et al. Enhancement of GSM Security Using Elliptic Curve Cryptography Algorithm; 2012 Third International Conference on Intelligent Systems Modelling and Simulation, 639-644. 